\title{
Análise de desempenho de handover em rede heterogênea usando MIH
}

\author{
Marco Antonio Simões Teixeira ${ }^{1}$, Alexandre Veloso de Matos $^{2}$, Mário Ezequiel \\ Augusto $^{2}$ \\ ${ }^{1}$ Engenharia Elétrica e Informática Industrial \\ Universidade Federal Tecnológica do Paraná (UTFPR) - Curitiba - PR - Brasil \\ ${ }^{2}$ Departamento de Sistemas de Informação \\ Universidade do Estado de Santa Catarina (UDESC) - São Bento do Sul - SC - Brasil \\ mantonio.t9l@gmail.com, \{alexandre.matos, mario.augusto\}@udesc.br
}

\begin{abstract}
Nowadays mobile devices have more than one network interface, in a way that they can access the Internet using many wireless network technologies. However, in this heterogeneous environment, handovers cause data and connection losses. IEEE proposed IEEE 802.21 protocol (MIH), an interlayer architecture to assist heterogeneous handovers and to minimize packet loss. One of its advantages is that it can preview the need of a handover, warning higher layers about the event. This paper presents results of simulations of a wireless heterogeneous environment where we analised the benefits of the Link_Going_Down event of IEEE 802.21. The protocol showed significant results related to throughput and handover delay.
\end{abstract}

Resumo. Atualmente os dispositivos móveis possuem várias interfaces de redes, podendo acessar a Internet usando várias tecnologias de rede sem fio. Porém, neste ambiente heterogêneo, as trocas de rede ocasionam perda de dados e de conexão. O IEEE propôs o protocolo IEEE 802.21 (MIH), uma arquitetura inter-camadas para auxiliar na troca de redes de tecnologias diferentes com a intenção de minimizar a perda de pacotes. Uma das vantagens é que ela pode prever a necessidade de uma troca de rede e avisar as camadas superiores sobre o evento. Este artigo apresenta os resultados da simulação de um ambiente heterogêneo sem fio onde nós analisamos os benefícios do evento Link_Going_Down do IEEE 802.21. O protocolo apresentou resultados significativos relacionados à vazão e ao tempo de handover.

\section{Introdução}

As tecnologias de comunicação e transmissão de dados estão evoluindo bastante. $\mathrm{Na}$ última milha, é crescente o uso do meio sem fio para transmitir e receber dados, através de tecnologias como Wi-Fi e rede celular (3G e $4 \mathrm{G})$. Em paralelo com o avanço das tecnologias sem fio, tem-se um avanço também nos dispositivos capazes de usufruir desses serviços. Tablets, smartphones, e outros dispositivos cada vez menores e mais potentes, possuem a capacidade para se conectar a redes sem fio, e a maioria possui a capacidade de se conectar em mais de uma tecnologia, como Wi-Fi e a telefonia móvel. 
Os aplicativos e serviços também evoluíram e passaram a depender mais do acesso à Internet. Outras aplicações surgiram, como o caso da aplicação VoIP (Voz sobre IP), a qual nós utilizamos como caso de uso nesse trabalho. O serviço de VoIP tem como objetivo estabelecer uma ligação de voz entre dois dispositivos, utilizando a Internet e o protocolo IP. Qualquer degradação do sinal de rádio pode provocar perda de pacotes, o que pode acarretar em atrasos na fala, ou até mesma a perda da ligação. Quando o dispositivo do usuário realiza uma troca de rede, a tendência é perder mais pacotes, principalmente se a troca ocorrer entre tecnologias diferentes ou redes diferentes. Este procedimento de troca de rede é denominado de handover.

Para tornar o handover suave (seamless) e diminuir o tempo de troca e a perda de pacotes, o IEEE criou um protocolo denominado IEEE 802.21 (2009) ou Media Independent Handover (MIH). Esse protocolo possui regras e normas para serem adotadas ao se praticar a troca entre redes, seja entre mesma tecnologia (handover horizontal) como, por exemplo, entre Wi-Fi e Wi-Fi, seja entre tecnologias diferentes (handover vertical) como Wi-Fi e rede celular. O MIH é um protocolo intercamadas, o qual encaminha comandos, eventos e informações para camadas inferiores e superiores.

Como este protocolo não é implementado nos equipamentos de rede dos principais fabricantes, não é possível conhecer o desempenho real do MIH através de ambientes reais, ou seja, não se sabe o quanto este protocolo pode melhorar as trocas de rede. Diversos pesquisadores têm realizado simulações do MIH para analisar seus benefícios.

Esse trabalho estudou, através de simulação, o quanto o IEEE 802.21 pode ajudar no problema de perda de dados ao se trocar de rede entre tecnologias distintas (handover heterogêneo ou vertical), mais especificamente, entre redes Wi-Fi e UMTS (rede celular 3G), embora também tenha sido analisado um handover homogêneo (ou horizontal), entre mesma tecnologia. Nós analisamos o uso do evento "Link Going Down” do MIH. Este evento avisa a aplicação que a conexão de rede atual será perdida.

Para realizar a simulação, nós utilizamos um simulador de redes comumente usado em pesquisas científicas, o simulador NS-2 (2015). Esse simulador possibilita virtualizar casos reais de transmissão e envio de dados. Para simular o MIH, nós acrescentamos o módulo NIST (2014) ao NS-2.

Os resultados demonstraram quanto o IEEE 802.21 pode otimizar as trocas de rede. Houve uma redução significativa na perda de dados e, consequentemente, aumento da vazão. A quantidade de pacotes perdidos durante a troca diminuiu em 98,4\%.

Além desta introdução, a seção 2 apresenta o protocolo IEEE 802.21 e seus serviços. Na seção 3 são apresentados alguns trabalhos relacionados ao gerenciamento de handovers e MIH. A seção 4 apresenta a metodologia utilizada neste trabalho, juntamente com o cenário heterogêneo simulado. A seção 5 apresenta os resultados de desempenho do MIH. As conclusões e trabalhos futuros encontram-se na seção 6.

\section{Media Independent Handover (MIH)}

O protocolo IEEE 802.21 (2009) tem como função facilitar o handover heterogêneo, principalmente entre tecnologias móveis, com o intuito de minimizar a perda de pacotes e aumentar o desempenho de redes. Ele deve ser implementado no nó móvel e também 
na estação base ou controlador. O protocolo cria uma camada intermediária entre a camada de redes e a camada de enlace, denominada MIHF - Media Independent Handover Function, à qual implementa três serviços: o Media Independent Command Service (MICS), o Media Independent Information Service (MIIS) e o Media Independent Event Services (MIES), como pode ser visto na Figura 1.

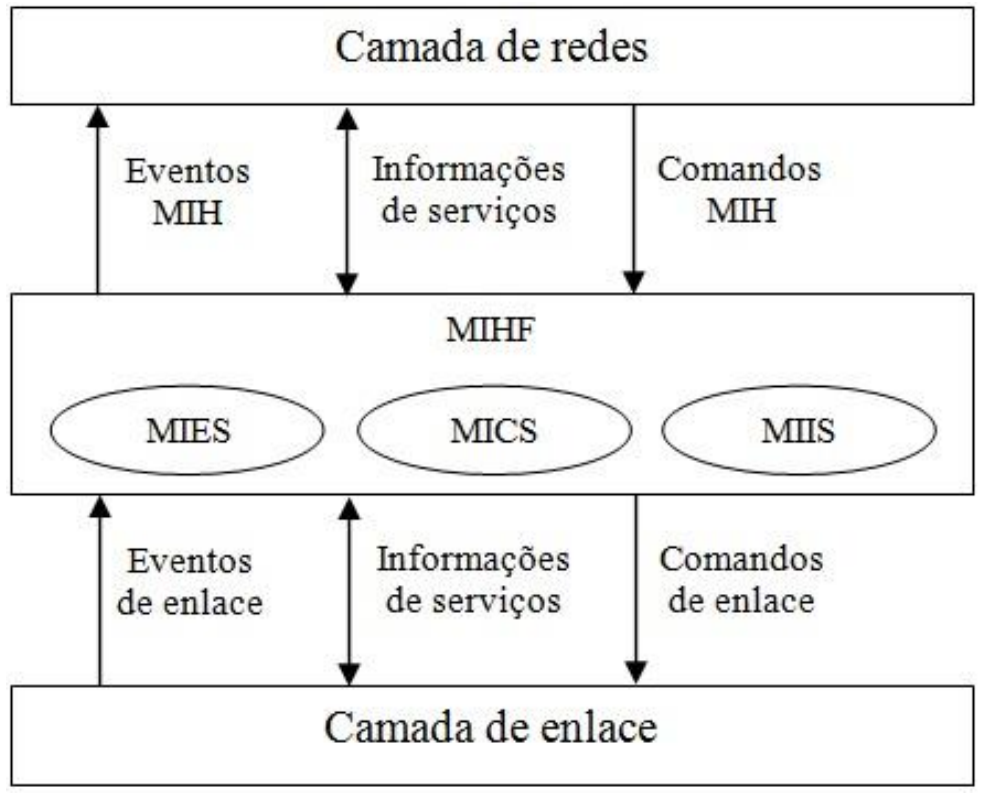

Figura 1 Arquitetura em camadas do IEEE $802.21(\mathrm{MIH})$

$\mathrm{Na}$ camada de rede e acima são implementados os MIH Users, programas que se inscrevem nos eventos do MIH, enviam comandos e recebem informações do serviço de informações (MIIS). O serviço de eventos (MIES) detecta eventos como, por exemplo, queda na conexão, nova rede detectada, degradação do sinal, entre outros, e alerta os $\mathrm{MIH}$ Users inscritos naquele evento. O serviço de informações (MIIS) fornece informações sobre as redes disponíveis para os MIH Users. O serviço de comandos (MICS) possibilita o envio de comandos entre as camadas do MIH como, por exemplo, inscrição em eventos e configuração de limites (thresholds).

Um dos eventos gerados pelo MIH é o Link_Going_Down, o qual avisa que as condições do enlace estão se degradando e a perda de conexão é iminente. Desta forma, um MIH User (gerenciador de conexões, por exemplo) fica sabendo antecipadamente que a conexão será perdida. Assim, prepara a troca de rede antes que ela ocorra, diminuindo o tempo de handover e a quantidade de pacotes perdidos. Os efeitos deste evento são analisados neste trabalho.

\section{Trabalhos relacionados}

Em um trabalho anterior (Augusto, 2012), nós realizamos simulações de handovers homogêneos, ou seja, entre redes utilizando a mesma tecnologia de acesso. Foram medidos o número de handovers, a quantidade de bytes recebidos, e quanto o usuário pagou pelo acesso. O ambiente era formado de várias antenas Wi-Fi e não foi utilizado o protocolo IEEE 802.21 devido à homogeneidade do ambiente. 
Relacionado ao trabalho atual, vários autores também simularam o IEEE 802.21 em um ambiente heterogêneo. Iqbal(2011) faz uso do IEEE 802.21 para a troca entre tecnologias de rede, utilizando como instrumento de testes o simulador de redes NS-2 com o pacote NIST (2014). Nos testes, os autores comprovam a eficiência do protocolo com handover heterogêneo, onde a vazão aumentou em 49,8\%. Porém, o ambiente analisado continha redes Wi-Fi e WiMAX. Outro trabalho parecido envolvendo cenário Wi-Fi e WiMAX é encontrado em Lim(2009).

Santos (2011) analisou o handover heterogêneo entre redes WiMAX e UMTS usando IP móvel e MIH através do simulador NS-2. Os autores analisaram a vazão, o atraso e o jitter médio, e compararam os resultados com os requisitos de Qualidade de Serviço especificados pelo 3GPP (3rd Generation Partnership Project).

Em Machan (2008), os autores simulam um cenário com antenas Wi-Fi e UMTS e comparam a utilização do MIH versus a não utilização. Os autores se concentram apenas na troca da rede Wi-Fi para a UMTS, medindo perda de pacotes e tempo perdido com a troca de rede. Os autores utilizaram o simulador NS-2 para a análise. Nosso trabalho analisou a troca em ambos os sentidos, além do handover homogêneo Wi-Fi para Wi-Fi.

Alguns trabalhos apresentam esquemas para otimização de handover heterogêneo utilizando MIH, como proposto por Augusto (2013), Wu (2009), Cicconetti (2011) e também fazem a análise de desempenho da utilização do protocolo.

Algumas implementações do IEEE 802.21 estão disponíveis. Além da implementação do NIST (2014) para o simulador NS-2, Corujo (2011) apresenta o ODTONE, uma implementação do protocolo que tem código-fonte aberto e é independente de sistema operacional. Outra implementação é a OpenMIH de Lopez (2009).

Para diferenciar as trocas de rede entre tipos diferentes, Mapp (2009) classifica os handovers entre imperativos e alternativos. Handovers imperativos são obrigatórios para evitar queda severa de desempenho ou queda na conexão, e do tipo alternativo ocorrem por opção (preço, incentivos, contexto, serviços oferecidos ou preferência do usuário). Mapp também classifica as trocas entre hard handover, quando a conexão atual é finalizada antes do dispositivo se conectar a outra rede, e soft handover, quando a conexão atual é finalizada após o dispositivo se conectar a outra rede. Nós utilizamos esta classificação para melhor comparar as trocas realizadas nas simulações.

\section{Metodologia e cenário analisado}

Nós realizamos a simulação utilizando-se o simulador de redes NS-2 (2015), juntamente com a implementação do IEEE 802.21 (MIH) e da tecnologia UMTS desenvolvida pelo NIST (2014). As tecnologias de redes escolhidas para realizar a simulação foram Wi-Fi e UMTS, devido à sua atual predominância de acesso a dados no Brasil (Opensignal, 2015).

O cenário é apresentado na Figura 2. As duas antenas Wi-Fi (WiFi1 e WiFi2) estão instaladas ao longo de uma avenida nas posições 100 metros e 180 metros, respectivamente, com raio de alcance de 50 metros. Desta forma, tem-se uma sobreposição de 20 metros entre ambas, viabilizando-se assim a análise de um hard 
handover homogêneo imperativo. O nó P2r é o roteador controlador das antenas Wi-Fi. O sinal da antena UMTS (Base Station) cobre todo o cenário. Os nós RNC, SGSN e GGSN são, respectivamente, Radio Network Controller, Serving GPRS Support Node, e Gateway GPRS Support Node do domínio UMTS. As duas tecnologias da forma como estão posicionadas no cenário, viabilizam um soft handover heterogêneo alternativo (UMTS $\rightarrow$ WiFi1) e um soft handover heterogêneo imperativo (WiFi2 $\rightarrow$ UMTS), além de um hard handover homogêneo imperativo (WiFi1 $\rightarrow$ WiFi2). As velocidades dos enlaces estão anotadas na figura.

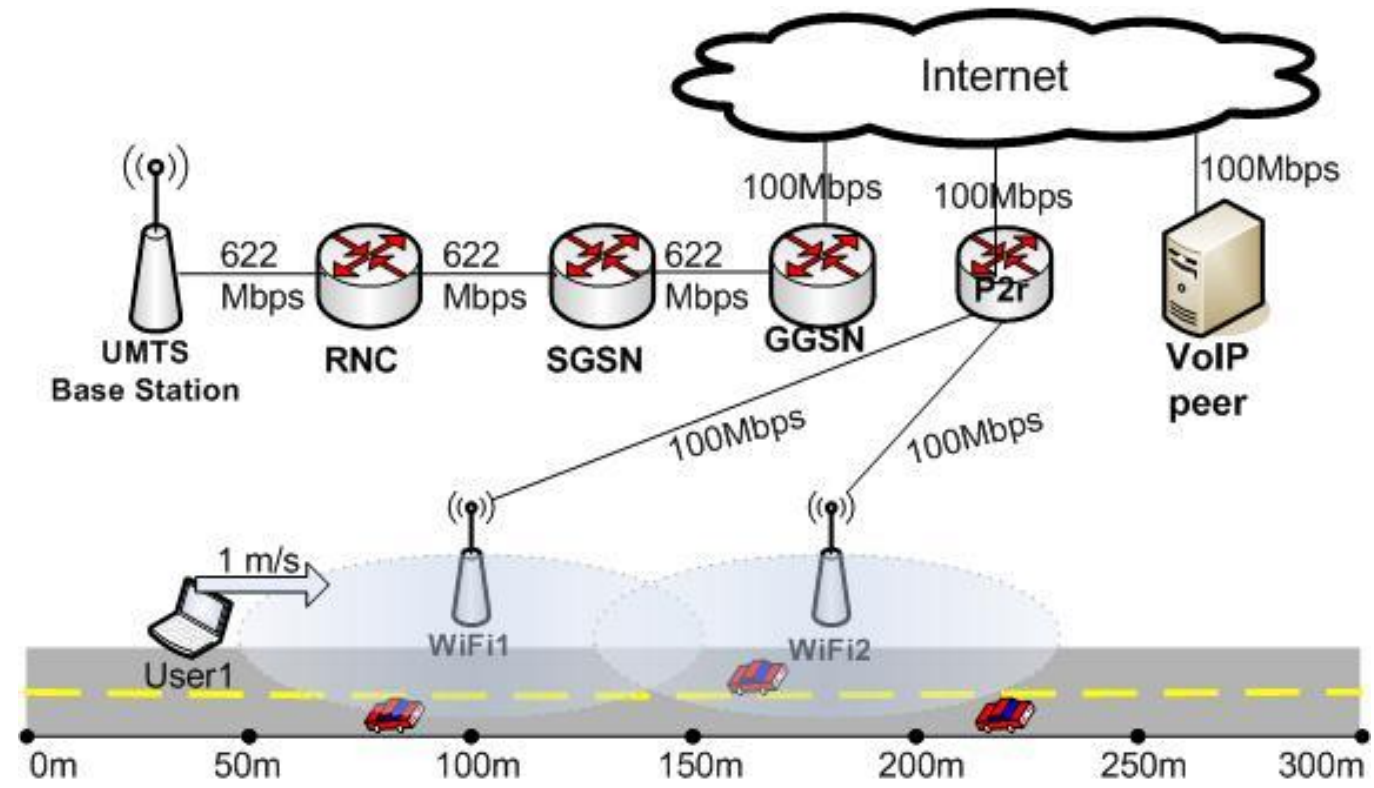

Figura 2 Cenário heterogêneo analisado

Um usuário móvel (User1) percorre a avenida a uma velocidade de $1 \mathrm{~m} / \mathrm{s}$, simulando uma pessoa caminhando, saindo da posição 40 metros aos 10 segundos de simulação até a posição 250 metros, passando pelas três antenas citadas anteriormente. O tempo total de simulação foi de 240 segundos. Neste percurso, o nó móvel primeiramente se conecta à antena UMTS, e em seguida conecta-se à antena WiFil, depois conecta-se à antena WiFi2, e quando perde o sinal desta última, volta a trafegar os dados pela antena UMTS.

Nós simulamos um tráfego VoIP entre o nó VoIP peer e o usuário móvel (User1). Os pacotes gerados foram do tipo UDP a uma taxa constante de bits de $64 \mathrm{kbps}$ (codec G.711). O tráfego teve início juntamente com a movimentação do nó móvel aos 10 segundos, e finalizou após o nó móvel parar de se mover, aos 240 segundos. No momento que este tráfego se inicia, o dispositivo já se encontra conectado à rede UMTS. Durante toda a movimentação do nó móvel, o tráfego estava fluindo. Para possibilitar a continuidade do tráfego após a troca de rede, nós utilizamos o protocolo Mobile IPv6 (Perkins, 2011).

\section{Resultados}

Nós realizamos simulações comparando a geração ou não do evento Link Going Down (LGD), comentado na Seção 2. Quando o evento LGD não estava ativado, a quantidade total de bytes recebidos pelo usuário móvel foi de $1663 \mathrm{~KB}$ (1703520 bytes) em um 
período de quase 4 minutos (230 segundos) trafegando o fluxo VoIP. Na simulação em que o evento LGD estava ativado, a quantidade de bytes recebidos aumentou para 1801 KB (1844960 bytes) no mesmo período, ou seja, um acréscimo de 8,3\%.

O acréscimo na quantidade total de bytes em si pode não ser tão significativo. Porém, considerando a aplicação em questão (VoIP), com suas exigências de Qualidade de Serviço, em dois dos três handovers na simulação em que nós não utilizamos o evento LGD, a conexão VoIP estaria comprometida. Os gráficos de vazão são apresentados na Figura 3.

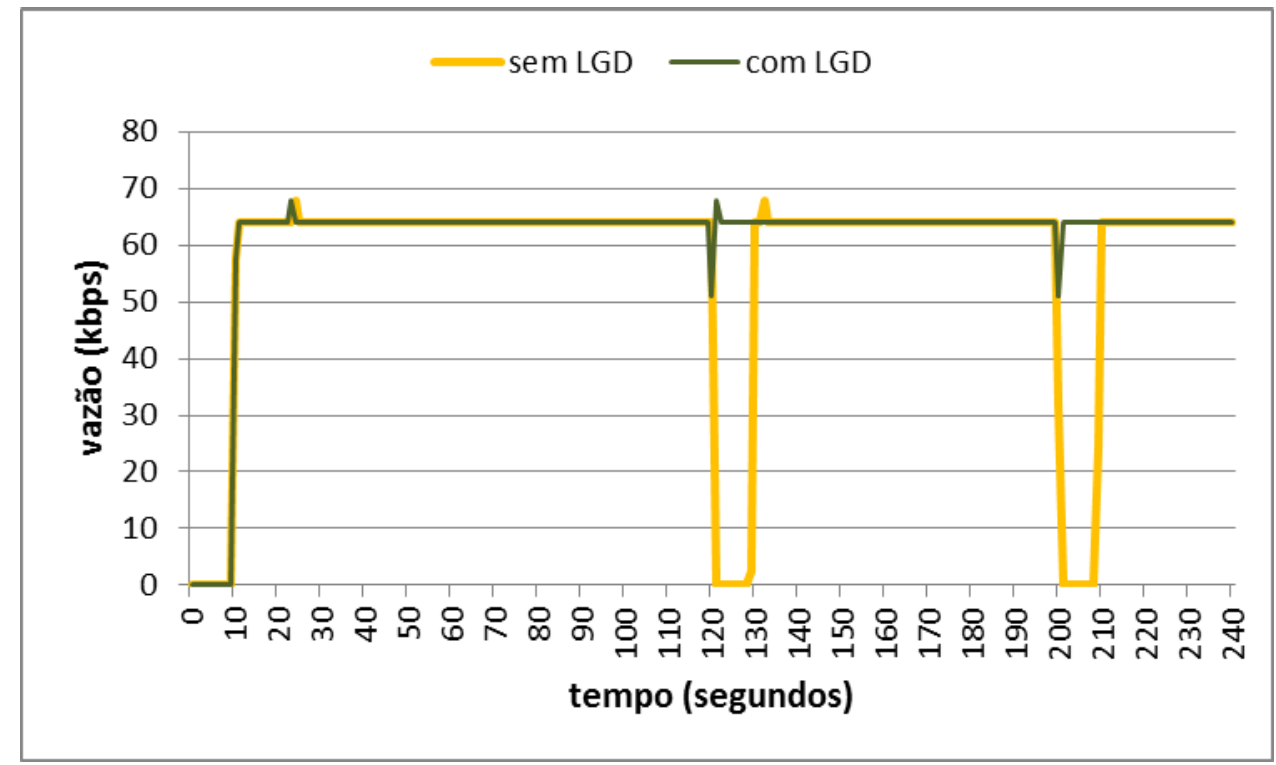

Figura 3 Gráfico dos resultados de vazão

$\mathrm{O}$ eixo $\mathrm{X}$ na Figura 3 refere-se ao tempo de simulação em segundos e o eixo $\mathrm{Y}$ refere-se à vazão do tráfego VoIP em kbps. Os três handovers ocorreram aproximadamente aos 22, 120 e 200 segundos. Estas três trocas são detalhadas a seguir.

\subsection{Soft handover heterogêneo alternativo (UMTS $\rightarrow$ WiFi1 aos 22 segundos)}

No gráfico da Figura 3, pode-se notar o fluxo VoIP iniciando aos 10 segundos de simulação. Neste momento o nó móvel já se encontrava conectado à antena UMTS. Neste mesmo momento, o nó móvel inicia sua caminhada a uma velocidade de $1 \mathrm{~m} / \mathrm{s}$. Aproximadamente aos 22 segundos de simulação, ocorre a troca entre a antena UMTS para a antena WiFi1. Enquanto os dados ainda trafegavam pela rede UMTS, o dispositivo detectou o sinal da antena WiFil e a conexão foi estabelecida, portanto troca do tipo soft handover heterogêneo. Como o dispositivo realizou a troca por uma questão de preferência, pois a implementação do simulador NS-2 prioriza as antenas Wi-Fi devido à maior velocidade de transmissão, o handover é classificado como sendo do tipo alternativo.

Em ambos os casos, sem LGD e com LGD, o tempo de handover foi zero pois o nó móvel conectou à rede Wi-Fi enquanto ainda trafegava dados normalmente pela interface UMTS. Consequentemente, a perda de pacotes também foi zero. 


\subsection{Hard handover homogêneo imperativo (WiFi1 $\rightarrow$ WiFi2 aos 120 segundos)}

Aproximadamente aos 120 segundos, a interface Wi-Fi perde sinal da antena WiFil pois já estava a 50 metros de distância, obrigando a interface a conectar-se com outra antena. Consultando as mensagens de log do simulador, é possível acompanhar cada passo do processo de handover. O processo é detalhado a seguir.

No caso da simulação sem LGD, aos 120 segundos a interface Wi-Fi perde sinal com a antena WiFil. Aos 128 segundos o prefixo Mobile IP da rede WiFil expira e o dispositivo inicia processo de scanning, ou seja, procura outras antenas disponíveis. Aos 129 segundos a interface Wi-Fi recebe resposta ao escaneamento da antena WiFi2 e a conexão é estabelecida. Aos 131 segundos, o agente MIPv6 recebe acknowledgement para redirecionamento do fluxo VoIP pela antena WiFi2. O tempo total sem recebimento de dados foi de 11,1 segundos. Neste intervalo de tempo sem receber dados, a quantidade de pacotes perdidos em função da troca de rede foi de 445 pacotes.

No caso da simulação com LGD, antes da interface perder o sinal da WiFi1, aos 117 segundos o protocolo MIH gerou o evento Link Going Down. Este evento se repete a cada 0,02 segundos e, a cada repetição, é gerada uma probabilidade de perda de conexão que inicia em 0\%. Quando esta probabilidade alcança 81\%, aos 119 segundos, a interface inicia processo de scanning. Logo em seguida já recebe resposta da antena WiFi2. Com isso a interface desconecta da WiFi1 e conecta-se à WiFi2. Aos 120 segundos, o agente MIPv6 recebe acknowledgement para redirecionamento do fluxo VoIP pela antena WiFi2. O tempo de handover foi de 0,8 segundos. Devido ao menor tempo para realizar a troca, a quantidade de pacotes perdidos diminuiu para apenas 7 pacotes.

\subsection{Soft handover heterogêneo imperativo (WiFi2 $\rightarrow$ UMTS aos 200 segundos)}

Aproximadamente aos 200 segundos, a interface Wi-Fi perde sinal da antena WiFi2 pois já estava a 50 metros de distância. No caso da simulação sem LGD, aos 199 segundos a interface Wi-Fi perde sinal com a antena WiFi2 e alguns pacotes começam a ser perdidos. Aos 208 segundos o prefixo da rede WiFi2 expira, o dispositivo inicia processo de scanning e o agente MIPv6 recebe acknowledgement para redirecionamento do fluxo VoIP pela antena UMTS. O processo de scanning continua até o final da simulação, sem obter resposta de antenas Wi-Fi.

No caso da simulação com LGD, aos 197 segundos o protocolo MIH gerou o evento Link Going Down, com probabilidade de perda de conexão incialmente de $0 \%$. Aos 199 segundos, a probabilidade alcança $81 \%$ e o agente de handover do MIH decide redirecionar o fluxo VoIP para a interface UMTS. No mesmo segundo, o agente MIH gera comando para iniciar scanning e o agente MIPv6 recebe acknowledgement para redirecionamento do fluxo VoIP pela antena UMTS. Logo em seguida, o evento Link Going Down indica probabilidade de $92 \%$ de perda de conexão. Com isso, a conexão Wi-Fi com a antena WiFi2 é derrubada. O processo de scanning continua até o final da simulação e o fluxo VoIP continua pela interface UMTS.

Em ambos os casos, sem e com LGD, o tempo de handover foi zero pois a interface UMTS já estava conectada. Porém, na simulação sem LGD o fluxo VoIP ficou 8,9 segundos sem recebimento de dados pois o handover demorou a ser percebido pela 
camada de rede, apenas quando houve a expiração do prefixo é que o fluxo foi redirecionado de interface. Na simulação com LGD, como houve a notificação do evento Link Going Down, o dispositivo se adiantou à perda da conexão e redirecionou o fluxo para a interface UMTS antecipadamente, evitando consideravelmente a perda de pacotes. Neste handover, na simulação sem LGD, um total de 453 pacotes foram perdidos. Com a geração do evento Link Going Down, esta quantidade caiu para apenas 7 pacotes.

\subsection{Repetição das simulações}

O simulador NS-2 utiliza geração de números aleatórios, cuja "semente" (seed) foi inicialmente fixada no valor 2 para ambas as simulações (com e sem LGD) para permitir uma comparação mais confiável entre ambos. Após a análise, comparação e geração dos gráficos de vazão, nós realizamos novamente as simulações, porém, utilizando seed aleatória. As simulações sem LGD resultaram em um total de bytes recebidos de 1699520, 1728320 e 1713600 bytes. Ou seja, o valor inicial 1703520 bytes ficou dentro do intervalo destes outros valores. As simulações com LGD resultaram na mesma quantidade de bytes recebidos que a inicial (1844960 bytes), provavelmente porque a troca é feita antes de haver depreciação significativa do sinal.

Os gráficos de vazão gerados nestas últimas simulações são parecidos com os gráficos gerados inicialmente, com os dois vales aos 120 e 200 segundos, em que a segunda e terceira troca foram feitas.

\section{Conclusões e trabalhos futuros}

Atualmente, a utilização de dispositivos móveis com várias interfaces de rede e a oferta de redes sem fio de diferentes tecnologias demanda o aperfeiçoamento das trocas de rede para possibilitar que o usuário aproveite este cenário para permanecer sempre conectado da melhor forma possível (Always Best Connected). O protocolo IEEE 802.21 (MIH - Media Independent Handover) tem como objetivo possibilitar as trocas de rede entre domínios diferentes e, principalmente, entre tecnologias de acesso diferentes.

O evento Link Going Down do IEEE 802.21 ocorre quando a interface de rede percebe que a força do sinal está se degradando, e avisa as camadas superiores (módulo de gerenciamento de mobilidade e aplicativos) que a conexão será perdida. Com isso, os aplicativos podem se antecipar ao handover, preparando a troca de rede antes de haver perdas significativas de dados.

Este trabalho simulou a utilização do evento Link Going Down em um cenário heterogêneo envolvendo redes Wi-Fi e UMTS (rede celular 3G), na qual um usuário móvel percorre uma trajetória passando por algumas antenas e realizando handovers. As simulações mostraram como o protocolo IEEE 802.21 pode auxiliar nas trocas de rede e também o quanto o evento Link Going Down pode otimizar estas trocas. Houve uma redução significativa na perda de dados e, consequentemente, aumento da vazão, evitando desta forma que um fluxo VoIP fosse interrompido. A grande vantagem deste evento é que os aplicativos e/ou um módulo gerenciador de handover pode antecipar a conexão com outra antena, diminuindo o tempo de troca. 
Neste trabalho, o dispositivo procurou por redes disponíveis próximas ao usuário, quando necessário, através de scanning. Os autores sugerem, como trabalhos futuros, a utilização do serviço de informações (MIIS) do IEEE 802.21 e a implementação de alguma política de decisão de acesso em um cenário com vários provedores. Também, utilizando as implementações disponíveis do MIH, citadas na seção Trabalhos Relacionados, é possível montar um testbed com várias antenas e o MIH sendo executado no nó móvel e na rede.

\section{Agradecimentos}

Os autores agradecem à Fundação de Amparo à Pesquisa e Inovação do Estado de Santa Catarina - FAPESC pelo apoio financeiro por meio do Termo de Outorga $\mathrm{N}^{\mathrm{o}}$ 2014TR3656, Edital Chamada Pública FAPESC No 04/2014 - Apoio à infraestrutura para grupos de pesquisa da UDESC.

\section{Referências}

Augusto, M. e Moreira, E. S. (2012), "Access on Demand on Wi-Fi Networks: the Impact of Dynamic Switching of Providers for the Mobile User", Workshop em Desempenho de Sistemas Computacionais e de Comunicação (Wperformance 2012), Anais do XXXII Congresso da Sociedade Brasileira de Computação, Julho.

Augusto, M. E., Vanni, R. M. P., Guardia, H. C., Aiash, M., Mapp, G. e Moreira, E. S. (2013), "MYHand: a Novel Architecture for Improving Handovers in NGNs", The Ninth Advanced International Conference on Telecommunications - AICT, Roma. Proceedings of the Advanced International Conference on Telecommunications, v. 1. p. 211-218, Junho.

Cicconetti, C., Galeassi, F., e Mambrini, R. (2011), "A software architecture for network-assisted handover in IEEE 802.21", Journal of Communications, vol. 6, no. 1, pp. 44-55.

Corujo, D., Guimaraes, C., Santos, B. e Aguiar, R. L. (2011), "Using an open-source IEEE 802.21 implementation for network-based localized mobility management", IEEE Communications Magazine, vol. 49, no. 9, pp. 114-123, doi: 10.1109/MCOM.2011.6011742, Setembro.

IEEE 802.21 Institute of Electrical and Electronics Engineers (2009), "IEEE Standard for Local and Metropolitan Area Networks - Part 21: Media Independent Handover Services, IEEE Std 802.21-2008”, DOI: 10.1109/IEEESTD.2009.4769367, Janeiro.

Iqbal, F. e Javed, M. Y. (2011), "Performance Analysis of MIH based Multihoming Approach of Vertical Handover in Heterogeneous Wireless Networks". International Journal of Future Generation Communication \& Network, Vol. 4 Issue 4, p103, Dezembro.

Lim, S., Kim, D., Suh, Y. e Won, J. (2009), "Implementation and performance study of IEEE 802.21 in integrated IEEE 802.11/802.16e networks", Computer Communications, pp. 134-143.

Lopez, Y. e Robert, E. (2009), "OpenMIH, an Open-Source Media-Independent Handover Implementation and Its Application to Proactive pre-Authentication", 
Mobile Networks and Management: First International Conference, MONAMI 2009, pp. 14-25, DOI 10.1007/978-3-642-11817-3_2, Outubro.

Machan, P., Swewin, S., e Wozniak, J. (2008), "Performance of mobility support mechanisms in a heterogeneous UMTS and IEEE 802.11 network offered under the IEEE 802.21 standard", International Conference on Information Technology.

Mapp, G., Shaikh, F., Aiash, M., Vanni, R.M. P., Augusto, M. e Moreira, E. S. (2009), "Exploring efficient imperative handover mechanisms for heterogenous wireless", Proceedings of the 12th International Conference on Network-Based Information Systems NBiS, p. 286-291, Agosto.

NIST - National Institute of Standards and Technology (2014), "Seamless and Secure Mobility Tool Suite (NIST Mobility)", Disponível em http://www.nist.gov/ctl/wireless-networks/ssm_tools.cfm, Outubro.

NS-2 Network Simulator (2015), Disponível em http://nsnam.isi.edu/nsnam, Outubro.

OpenSignal (2015), OpenSignal Site, Disponível em: <http://opensignal.com>, Dezembro.

Perkins, C. (2011), “Mobility Support in IPv6 - RFC6275”, ISSN 2070-1721.

Santos, W. P., Reis, S. O., Nogueira, R. S. e Figueiredo, F. L. P. D. (2011), "Modelo de handover vertical suave entre redes WiMAX e UMTS", XVI Workshop de Gerência e Operação de Redes e Serviços (WGRS), Campo Grande, Mato Grosso do Sul.

Wu, J., Yang, S., e Hwang, B. (2009), “A terminal-controlled vertical handover decision scheme in IEEE 802.21-enabled heterogeneous wireless networks", International Journal of Communication Systems, vol. 22, no. 7, pp. 819-834, Julho. 